\title{
THERMOELASTIC INSTABILITY IN PLANAR SOLIDIFICATION
}

\author{
NAI-YI LI ${ }^{\dagger}$ and J. R. BARBER \\ Department of Mechanical Engineering and Applied Mechanics, University of Michigan, Ann Arbor, \\ MI 48109-2125, U.S.A.
}

(Received 22 August 1990; and in revised form 27 June 1991)

\begin{abstract}
A linear perturbation method is used to examine the stability of a unidirectional solidification problem in which a liquid, initially at the melting temperature, becomes solidified by heat transfer across a pressure-dependent thermal contact resistance to a plane mold. The contact pressure will be influenced by thermal distortion in response to the instantaneous temperature field in the solidified shell. The heat transfer and thermal stress problems are therefore coupled through the boundary conditions.

The temperature and stress fields are assumed to consist of a unidirectional component and a small spatially-sinusoidal perturbation which can vary with time. Analysis of the thermoelastic problem for the solidified shell leads to an ordinary differential equation relating the perturbation in heat flux at the mold/casting interface and the corresponding perturbation in contact pressure. A second equation relating the same two variables is obtained by linear perturbation of the relation for heat conduction across the thermal contact resistance. These are then reduced to a single equation which is solved numerically. The results show that a small initial perturbation will grow substantially during the solidification process if the thermal contact resistance is very sensitive to pressure.
\end{abstract}

\section{INTRODUCTION}

During the casting process, an initially liquid mass of material is caused to solidify by reducing its temperature below the melting point by heat transfer from its surfaces, which are in contact with a surrounding mold. There will be some thermal contact resistance at the mold/solid interface, since the surfaces are rough on the microscopic scale and generally carry adherent contaminant and oxide films. This thermal contact resistance has formed the subject of many investigations, both experimental $[1,2]$ and theoretical $[3,4]$ and it is generally agreed that it is very sensitive to contact pressure, principally because, being rough, the surfaces make contact only at the peaks of microscopic asperities and the number and size of these "actual contact areas" increases with load [5].

Initially, the contact pressure at the mold/casting interface will be determined by the hydrostatic pressure in the liquid, but as solidification proceeds, the temperature gradient through the solidified shell will induce thermoelastic distortion and hence influence the contact pressure. Ho and Pehlke $[6,7]$ deduced values of thermal contact conductance from temperature measurements during solidification and found that the conductance falls during the process. This effect is most probably attributable to shrinkage of the casting, resulting in a reduced contact pressure at the casting mold interface. If the initial hydrostatic pressure is insufficiently high, an air gap may develop at this interface, causing a substantial reduction in interface conductance $[6,8]$.

The heat transfer and thermal stress problems are therefore coupled through the boundary conditions. This coupled process is potentially unstable and is cited by Richmond and Huang [8] as a possible explanation for the long-wavelength perturbations which are sometimes observed in the nominally planar solidification front in unidirectional solidification $[9,10]$. Such perturbations are undesirable in the manufacturing process since the associated non-uniform thermal fields can cause cracks to develop during solidification [11].

Richmond et al. [12] recently developed an idealized analysis of this problem, in which a constant heat flux across the interface is taken to have a small prescribed spatiallysinusoidal perturbation. They found that the resulting thermal distortion increased the contact pressure in the regions where the heat flux is greatest. This indicates that the

${ }^{\dagger}$ Present address: ALCOA laboratories, Alcoa Center, PA 15069, U.S.A. 


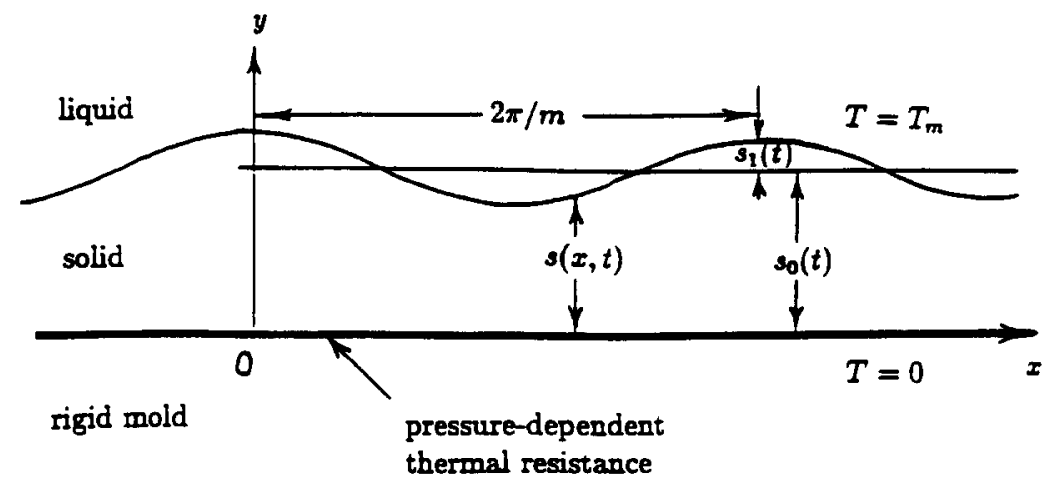

FIG. 1. Geometry of the system.

coupled problem has positive feedback, but it is not a conclusive demonstration of the plausibility of the proposed mechanism, since not all positive feedback systems are unstable.

In the present paper, we shall develop a linear perturbation solution of the coupled problem of Fig. 1, in which the heat flux at the mold/casting interface, $y=0$, is determined by a pressure-dependent contact resistance. In particular, we shall demonstrate that a range of long-wavelength perturbations are unstable if the contact resistance is sufficiently pressure-sensitive. We anticipate that those wavelengths with the greatest growth rate will dominate the behavior of the system if the initial perturbation is very small and spatially random. The analysis involves the solution of a heat-conduction problem with phase change, the determination of the instantaneous thermoelastic stress field associated with the resulting temperature field, and the coupling of these two calculations by perturbation of the heat flow across a pressure-dependent thermal contact resistance. Before formulating the problem, we first introduce some general concepts which will be needed in the solution of the thermoelastic problem.

\section{THE DEVELOPMENT OF RESIDUAL STRESS}

The determination of the stress field is complicated by the fact that material is continually being solidified while the solid is in a deformed state, so that the final cast product exhibits residual stress, even after the temperature has been reduced to zero. The simplest constitutive model for the process assumes that the material behaves elastically after solidification. This assumption is clearly very idealized, particularly in view of the fact that the yield strength of most materials falls rapidly when the temperature approaches the melting point, but it is adopted in the present paper in order to expose qualitative features of the problem in the simplest form. However, we might note that the newly-solidified material is deposited in a state of hydrostatic compression and will only gradually deviate from this condition at any given point as solidification proceeds. It therefore follows that the material nearest the melting temperature-i.e. that near to the melt/solid boundary-will experience comparatively little shearing stress.

The thermoelastic solution of the solidification problem is conveniently formalized by writing

$$
\sigma=\sigma^{\mathrm{P}}+\sigma^{\mathrm{H}}
$$

where $\sigma^{\mathrm{P}}$ represents a suitable particular thermoelastic solution corresponding to the temperature distribution, but without regard to the boundary conditions, and $\sigma^{\mathbf{H}}$ is a solution of the "homogeneous" problem with no thermal strains which introduces additional degrees of freedom to enable us to satisfy the mechanical boundary conditions.

The particular solution can be represented in terms of a thermoelastic displacement potential $\phi$ (see [13]) where

$$
\nabla^{2} \phi=\frac{E \alpha}{1-v} T
$$

$T$ describes the temperature field, and $E, \alpha, v$ are the Young's modulus, coefficient of thermal 
expansion and Poisson's ratio of the material, respectively. We assume that all the material constants are independent of temperature. The stress and displacement components can then be expressed in terms of $\phi$ using the relations [13]

$$
\begin{aligned}
\sigma_{x x}^{\mathrm{P}} & =-\frac{\partial^{2} \phi}{\partial y^{2}}-\frac{\partial^{2} \phi}{\partial z^{2}} \\
\sigma_{x y}^{\mathrm{P}} & =\frac{\partial^{2} \phi}{\partial x \partial y} \\
\sigma_{y y}^{\mathrm{P}} & =-\frac{\partial^{2} \phi}{\partial x^{2}}-\frac{\partial^{2} \phi}{\partial z^{2}} \\
\frac{E}{(1+v)} u_{y}^{\mathrm{P}} & =\frac{\partial \phi}{\partial y} .
\end{aligned}
$$

The homogeneous solution can be represented by an Airy stress function, $\Phi$, where

$$
\sigma_{x x}^{\mathrm{H}}=\frac{\partial^{2} \Phi}{\partial y^{2}} ; \quad \sigma_{x y}^{\mathrm{H}}=-\frac{\partial^{2} \Phi}{\partial x \partial y} ; \quad \sigma_{y y}^{\mathrm{H}}=\frac{\partial^{2} \Phi}{\partial x^{2}} .
$$

This formulation ensures that the resulting stress field satisfies the equilibrium equations.

During the process, material is continually being solidified while the solid is in a deformed state, it therefore follows that there is no convenient initial condition to describe the displacement field and hence the strains. The strains are therefore not required to satisfy the compatibility equation and hence $\Phi$ does not have to be biharmonic. However, since no inelastic strains can occur at any point after it has solidified, we conclude that the incompatible strains must be independent of time and hence

$$
\frac{\partial}{\partial t} \nabla^{4} \Phi=0
$$

The general solution of Eqn (5) can be decomposed into two parts, i.e. $\Phi=\Phi_{1}+\Phi_{2}$, where

$$
\frac{\partial \Phi_{1}}{\partial t}=0 ; \quad \nabla^{4} \Phi_{2}=0 .
$$

In physical terms, we can then interpret $\Phi_{1}$ as representing the residual stress, i.e. the stress which remains when the fully-solidified casting has been cooled to a uniform temperature and has traction-free boundaries (which is time-independent but not necessarily biharmonic) and $\Phi_{2}$ as the instantaneous compatible response of the solid to the changing temperature field. ${ }^{\dagger}$

An additional condition, analogous to a flow rule, is needed to determine the function $\Phi_{1}$. This is obtained from the assumption that there is no discontinuous change in the stress state of a particle as it passes from the liquid to the solid state. It therefore follows that the newly-solidified material is instantaneously in a state of hydrostatic compression [14].

\section{FORMULATION OF THE PROBLEM}

We now return to the problem of Fig. 1. To develop a fully-coupled solution, we adopt the following procedure. First, we investigate the temperature field (Section 3.1) and the stress field (Section 3.2) due to a prescribed sinusoidal perturbation in the heat flux at the casting/mold interface. In particular, we determine the relation between the prescribed heat flux and the perturbation in contact pressure at the casting/mold interface. Richmond's solution [12] is shown to be a special case of this relation in Section 3.3. Then, in Section 3.4, we obtain a second relation between the heat flux at the interface and the

\footnotetext{
${ }^{\dagger}$ The decomposition of a general function satisfying Eqn (5) into $\Phi_{1}, \Phi_{2}$ is not unique since an arbitrary timeindependent biharmonic function can be subsumed under either part.
} 
contact pressure by perturbing the relation for the heat flux across a pressure-dependent contact resistance. This leads to a pair of coupled ordinary differential equations, whose solution is discussed in Section 4.

\subsection{The heat conduction problem}

The temperature of the solid $T(x, y, t)$ must satisfy the heat conduction equation:

$$
\nabla^{2} T=\frac{1}{k} \frac{\partial T}{\partial t}
$$

and the boundary conditions

$$
\begin{aligned}
T(x, s, t) & =T_{m} \\
K \frac{\partial T}{\partial y}(x, s, t) & =L \rho \frac{\partial s}{\partial t}(x, t) \\
K \frac{\partial T}{\partial y}(x, 0, t) & =Q(x, t)
\end{aligned}
$$

where $y=s(x, t)$ defines the instantaneous position of the solid/melt boundary and we assume that the thermal diffusivity $k$, conductivity $K$ and density $\rho$ of the solid phase are constant and independent of temperature. Note that $L$ is the latent heat of fusion of the material.

Equation (8) states that there must be continuity of temperature at the solid/melt interface, while (9) defines an energy balance between the heat conducted away from the moving interface into the solid and the latent heat released during solidification. The heat flux at the casting/mold interface is prescribed by (10).

We consider the case in which the prescribed heat flux is of the form

$$
\begin{aligned}
Q(x, t) & =Q_{0}(t)+Q_{1}(t) \cos (m x) \\
Q_{0}(t) & \gg Q_{1}(t)
\end{aligned}
$$

where the second term defines a small sinusoidal perturbation on the uniform cooling rate, $Q_{0}(t)$. Since $Q_{1}(t) \ll Q_{0}(t)$, we anticipate a corresponding small sinusoidal perturbation in the thickness of the solidified layer, i.e.

$$
\begin{aligned}
s(x, t) & =s_{0}(t)+s_{1}(t) \cos (m x) \\
s_{0}(t) & \gg s_{1}(t) .
\end{aligned}
$$

We also assume that the amplitude of this perturbation is small in comparison with its wavelength (i.e. $s_{1} m \ll 1$ ), in which case the slope of the moving front $\partial s / \partial x \ll 1$. It then follows that the heat flux in the $x$-direction is negligible to first order. Finally, to simplify the analysis, we assume that the Stefan number $\varepsilon \ll 1[15]$. This is equivalent to the statement that the thermal capacity of the material is small in comparison with the latent heat. It then follows that Eqn (7) approximates Laplace's equation and in view of the condition, $\partial s / \partial x \ll 1$, that the temperature profile in the solid is linear in $y$. Using $(8),(10)$, we then have

$$
T(x, y, t)=T_{m}+\frac{Q(x, t)}{K}(y-s) .
$$

Substituting Eqn (13) into (9) we have

$$
\frac{\partial s(x, t)}{\partial t}=\frac{Q(x, t)}{\rho L}
$$

and hence from (11), (12), (14),

$$
Q_{0}(t)=L \rho \dot{s}_{0}(t) ; \quad Q_{1}(t)=L \rho \dot{s}_{1}(t)
$$

where $\left({ }^{\circ}\right)$ denotes differentiation with respect to time $t$. 
Substituting (11), (12) into (13), we have

$$
T(x, y, t)=T_{m}+\frac{\left[Q_{0}(t)+Q_{1}(t) \cos (m x)\right]}{K}\left[y-s_{0}(t)-s_{1}(t) \cos (m x)\right] .
$$

Separating periodic and uniform terms, dropping the second-order term in the small quantities, $Q_{1}(t), s_{1}(t)$, and using (15), we can express the temperature field in the solid in the form

where

$$
T(x, y, t)=T_{0}(y, t)+T_{1}(y, t) \cos (m x)
$$

$$
\begin{aligned}
& T_{0}(y, t)=T_{m}+\frac{L \rho \dot{s}_{0}(t)}{K}\left[y-s_{0}(t)\right] \\
& T_{1}(y, t)=\frac{-L \rho}{K}\left\{\dot{s}_{0}(t) s_{1}(t)+\dot{s}_{1}(t)\left[s_{0}(t)-y\right]\right\} \\
& T_{1}(y, t) \ll T_{0}(y, t)-T_{0}(0, t) .
\end{aligned}
$$

\subsection{Determination of the stress field}

We now determine the stress field, using the technique outlined in Section 2 above. For this unidirectional solidification problem, the mechanical boundary conditions are

$$
\begin{aligned}
\sigma_{x x}=\sigma_{y y} & =-p, \quad \sigma_{x y}=0 ; \quad y=s(t) \\
\sigma_{x y} & =0 \quad \forall t ; \quad y=0, \\
\dot{u}_{y} & =0 \quad \forall t ; \quad y=0,
\end{aligned}
$$

where $(20)$ indicates that the stress state at the solid/liquid interface is one of hydrostatic compression (see Section 2) and (21), (22) define frictionless contact conditions at the casting/mold interface. Notice that condition (22) can only be stated in terms of a time derivative since there is no reference state for the displacement.

In view of the form of the temperature field (17), we anticipate a stress field of the form

$$
\sigma(x, y, t)=\sigma_{0}(y, t)+\sigma_{1}(x, y, t)
$$

where $\sigma_{0}(y, t) \gg \sigma_{1}(x, y, t)$ and $\sigma_{1}(x, y, t)$ is a small perturbation on the stress field $\sigma_{0}(y, t)$ of the unidirectional or zeroth-order problem.

In particular, we anticipate that the contact pressure at the casting/mold interface, $P(x, t)\left[\equiv-\sigma_{y y}(x, 0, t)\right]$, will take the form

where

$$
P(x, t)=P_{0}(t)+P_{1}(t) \cos (m x)
$$

$$
\begin{aligned}
& P_{0}(t)=-\sigma_{y y 0}(0, t) ; \quad P_{1}(t) \cos (m x)=-\sigma_{y y 1}(x, 0, t) \\
& P_{0}(t) \gg P_{1}(t) .
\end{aligned}
$$

3.2.1. The zeroth-order solution. We first consider the zeroth-order field, corresponding to the temperature field $T_{0}(y, t)$ defined by Eqn (18). An appropriate displacement potential satisfying (2) is

$$
\phi_{0}=\frac{E \alpha}{1-v}\left\{\frac{1}{2}\left[T_{m}-\frac{L \rho \dot{s}_{0}(t)}{K} s_{0}(t)\right] y^{2}+\frac{L \rho \dot{s}_{0}(t)}{6 K} y^{3}\right\}
$$

from which Eqns (3) define the stress components

$$
\begin{aligned}
& \sigma_{x x 0}^{\mathrm{P}}=\frac{E \alpha}{1-v}\left\{-T_{m}+\frac{L \rho \dot{s}_{0}(t)}{K}\left[s_{0}(t)-y\right]\right\} \\
& \sigma_{x y 0}^{\mathrm{P}}=0 ; \quad \sigma_{y y 0}^{\mathrm{P}}=0 .
\end{aligned}
$$

To complete the solution, we must superpose a homogeneous stress field and assign the arbitrary constants to satisfy the boundary conditions (20)-(22). It is easily verified that this 
is achieved by the uniform biaxial field

$$
\sigma_{x x 0}^{\mathrm{H}}=\frac{E \alpha T_{m}}{1-v}-p ; \quad \sigma_{x y 0}^{\mathrm{H}}=0 ; \quad \sigma_{y y 0}^{\mathrm{H}}=-p
$$

so that the complete zeroth-order solution is

$$
\begin{aligned}
& \sigma_{x x 0}(y, t)=-p+\frac{E \alpha L \rho \dot{s}_{0}(t)}{(1-v) K}\left[s_{0}(t)-y\right] \\
& \sigma_{y y 0}(y, t)=-p ; \quad \sigma_{x y 0}(y, t)=0 .
\end{aligned}
$$

In particular, we have $P_{0}(t)=p$ from (25), (31).

3.2.2. The first-order solution. We now consider the thermoelastic problem corresponding to the temperature field $T_{1}(y, t) \cos (m x)$ [see Eqns (19), (21)]. A suitable particular solution can be obtained by assuming a potential in the form $\phi_{1}=g(y) \cos (m x)$ and using (2) to determine the function $g(y)$, with the result

where

$$
\phi_{1}=\frac{A}{m^{2}}\left[B(t)-\dot{s}_{1}(t) y\right] \cos (m x)
$$

$$
\begin{aligned}
A & =\frac{E \alpha L \rho}{(1-v) K} \\
B(t) & =\dot{s}_{0}(t) s_{1}(t)+\dot{s}_{1}(t) s_{0}(t) .
\end{aligned}
$$

The corresponding stress components from (3), (32)-(34) are

$$
\begin{aligned}
\sigma_{x x 1}^{\mathrm{P}} & =0 \\
\sigma_{y y 1}^{\mathrm{P}} & =A\left[B(t)-\dot{s}_{1}(t) y\right] \cos (m x) \\
\sigma_{x y 1}^{\mathrm{P}} & =\frac{A \dot{s}_{1}(t)}{m} \sin (m x) .
\end{aligned}
$$

Following Section 2, we represent the homogeneous solution of the first-order problem in terms of an Airy stress function $\Phi$. In view of Eqns (5), (6), $\Phi$ will involve four linearlyindependent biharmonic functions of the spatial coordinates with time-dependent coefficients and one time-independent function. The appropriate form is

$$
\Phi=\left\{\left[a_{1}(t)+a_{2}(t) y\right] \cos (m y)+\left[a_{3}(t)+a_{4}(t) y\right] \sinh (m y)+f(y)\right\} \cos (m x)
$$

in which the time-dependent coefficients $a_{1}(t), a_{2}(t), a_{3}(t), a_{4}(t)$, and time-independent function $f(y)$ (which corresponds to the residual stress) are to be determined from the mechanical boundary conditions corresponding to the first-order problem.

Using Eqns (1), (4), (35)-(37), (38), we can construct the complete stress field of the firstorder problem in the form

$$
\begin{aligned}
\sigma_{x x 1}(x, y, t)= & \left\{\left[a_{1}(t)+a_{2}(t) y+\frac{2 a_{4}(t)}{m}\right] \cosh (m y)\right. \\
& \left.+\left[a_{3}(t)+a_{4}(t) y+\frac{2 a_{2}(t)}{m}\right] \sinh (m y)+\frac{1}{m^{2}} \frac{\mathrm{d}^{2} f}{\mathrm{~d} y^{2}}\right\} m^{2} \cos (m x) \\
\sigma_{x y 1}(x, y, t)= & \left\{\frac{A \dot{s}_{1}(t)}{m^{3}}+\left[a_{1}(t)+a_{2}(t) y+\frac{a_{4}(t)}{m}\right] \sinh (m y)\right. \\
& \left.+\left[a_{3}(t)+a_{4}(t) y+\frac{a_{2}(t)}{m}\right] \cosh (m y)+\frac{1}{m} \frac{\mathrm{d} f}{\mathrm{~d} y}\right\} m^{2} \sin (m x) \\
\sigma_{y y 1}(x, y, t)= & \left\{\frac{A}{m^{2}}\left[B(t)-\dot{s}_{1}(t) y\right]-\left[a_{1}(t)+a_{2}(t) y\right] \cosh (m y)\right. \\
& \left.-\left[a_{3}(t)+a_{4}(t) y\right] \sinh (m y)-f(y)\right\} m^{2} \cos (m x) .
\end{aligned}
$$


As explained in Section 2 above, the strains due to these stress components are not required to be compatible, since there is no initial reference state, but the strain ratesobtained by substituting (39)-(41) into the thermoelastic constitutive relations and differentiating with respect to time-are compatible and can therefore be expressed as displacement derivatives and integrated to give the velocity components.

In particular, we obtain

$$
\begin{aligned}
\frac{-E}{(1+v)} \dot{u}_{y 1}(x, y, t)= & \left\{\frac{A \ddot{s}_{1}(t)}{m^{3}}+\left[\dot{a}_{1}(t)+\dot{a}_{2}(t) y-(1-2 v) \frac{\dot{a}_{4}(t)}{m}\right] \sinh (m y)\right. \\
& \left.+\left[\dot{a}_{3}(t)+\dot{a}_{4}(t) y-(1-2 v) \frac{\dot{a}_{2}(t)}{m}\right] \cosh (m y)\right\} m \cos (m x) .
\end{aligned}
$$

We now consider the boundary conditions corresponding to the first-order problem. Since the perturbation on the stress field is small, we can expand the stress field in the vicinity of the mean solid/melt interface position, $y=s_{0}(t)$, in the form of a Taylor series, in which case the first boundary condition in (20) can be written as

$$
\begin{aligned}
\sigma_{x x 0}\left(s_{0}, t\right) & +\frac{\partial \sigma_{x x 0}\left(s_{0}, t\right)}{\partial y} s_{1}(t) \cos (m x)+\frac{\partial^{2} \sigma_{x x 0}\left(s_{0}, t\right)}{\partial y^{2}} \frac{s_{1}^{2}(t) \cos ^{2}(m x)}{2 !}+\cdots \\
& +\left[\sigma_{x x 1}\left(x, s_{0}, t\right)+\frac{\partial \sigma_{x x 1}\left(x, s_{0}, t\right)}{\partial y} s_{1}(t) \cos (m x)+\cdots\right]=-p
\end{aligned}
$$

Separating periodic and uniform terms, dropping second- and higher-order and product terms in the small quantities, $\sigma_{x x 1}, s_{1}$, and using Eqn (30), we obtain the boundary condition for $\sigma_{x x 1}$ at $y=s_{0}(t)$, i.e.

$$
\sigma_{x x 1}\left(x, s_{0}(t), t\right)=A \dot{s}_{0}(t) s_{1}(t) \cos (m x)
$$

Applying the same procedure to the remaining boundary conditions in (20), we obtain

$$
\sigma_{x y 1}\left(x, s_{0}(t), t\right)=0 ; \quad \sigma_{y y 1}\left(x, s_{0}(t), t\right)=0 .
$$

Also from conditions (21), (22), we have

$$
\sigma_{x y 1}(x, 0, t)=0 ; \quad \dot{u}_{y 1}(x, 0, t)=0 .
$$

Applying the boundary conditions (46), using (40), (42), we can determine the time derivatives of the time-dependent coefficients, $\dot{a}_{2}(t)$ and $\dot{a}_{3}(t)$, i.e.

$$
\dot{a}_{2}(t)=0 ; \quad \dot{a}_{3}(t)=\frac{-A \ddot{s}_{1}(t)}{m^{3}} .
$$

We can therefore write

$$
a_{2}(t)=0 ; \quad a_{3}(t)=\frac{-A \dot{s}_{1}(t)}{m^{3}}
$$

without loss of generality, since constants of integration will lead to time-independent terms in $\Phi$ which can be subsumed under $f(y)$.

Substituting for the stress components from Eqns (39)-(41) into the remaining boundary conditions (44), (45), we obtain the equations

$$
\begin{aligned}
a_{1}(t) \cosh \left(m s_{0}(t)\right) & +a_{4}(t)\left[\frac{2}{m} \cosh \left(m s_{0}(t)\right)+s_{0}(t) \sinh \left(m s_{0}(t)\right)\right] \\
& +\frac{1}{m^{2}} f^{\prime \prime}\left(s_{0}(t)\right)=\frac{A}{m^{2}}\left[\dot{s}_{0}(t) s_{1}(t)+\frac{\sinh \left(m s_{0}(t)\right)}{m} \dot{s}_{1}(t)\right] \\
a_{1}(t) \sinh \left(m s_{0}(t)\right)+ & a_{4}(t)\left[\frac{1}{m} \sinh \left(m s_{0}(t)\right)+s_{0}(t) \cosh \left(m s_{0}(t)\right)\right] \\
+ & \frac{1}{m} f^{\prime}\left(s_{0}(t)\right)=\frac{-A \dot{s}_{1}(t)}{m^{3}}\left[1-\cosh \left(m s_{0}(t)\right)\right]
\end{aligned}
$$




$$
\begin{aligned}
a_{1}(t) \cosh \left(m s_{0}(t)\right)+ & a_{4}(t) s_{0}(t) \sinh \left(m s_{0}(t)\right)+f\left(s_{0}(t)\right) \\
& =\frac{A}{m^{2}}\left[\dot{s}_{0}(t) s_{1}(t)+\frac{\sinh \left(m s_{0}(t)\right)}{m} \dot{s}_{1}(t)\right]
\end{aligned}
$$

where we have used (48) to eliminate $a_{2}(t), a_{3}(t)$. These three equations must be satisfied for all values of $t$ and hence we can use them to eliminate $a_{1}(t), a_{4}(t)$. Defining $w=\tanh \left(m s_{0}(t)\right)$, we obtain

$$
\begin{aligned}
\frac{1}{2 m^{2}} & {\left[m s_{0}(t) w^{2}-w-m s_{0}(t)\right] f^{\prime \prime}\left(s_{0}(t)\right)+\frac{1}{m} f^{\prime}\left(s_{0}(t)\right) } \\
& -\frac{1}{2}\left[m s_{0}(t) w^{2}+w-m s_{0}(t)\right] f\left(s_{0}(t)\right) \\
= & -\frac{A}{m^{3}} \dot{s}_{1}(t)\left[1-\frac{1}{\cosh \left(m s_{0}(t)\right)}\right]-\frac{A}{m^{2}} \dot{s}_{0}(t) s_{1}(t) w
\end{aligned}
$$

which serves to determine the unknown residual stress function $f(y)$. Once $f(y)$ is known, we can recover $a_{1}(t), a_{4}(t)$ from Eqns (49), (51) in the form

$$
\begin{aligned}
a_{1}(t)= & \frac{1}{\cosh \left(m s_{0}(t)\right)}\left\{\frac{A}{m^{2}}\left[\dot{s}_{0}(t) s_{1}(t)+\frac{\sinh \left(m s_{0}(t)\right)}{m} \dot{s}_{1}(t)\right]\right. \\
& \left.-\frac{m s_{0}(t) w}{2}\left[f\left(s_{0}(t)\right)-\frac{1}{m^{2}} f^{\prime \prime}\left(s_{0}(t)\right)\right]-f\left(s_{0}(t)\right)\right\} \\
a_{4}(t)= & \frac{m}{2 \cosh \left(m s_{0}(t)\right)}\left[f\left(s_{0}(t)\right)-\frac{1}{m^{2}} f^{\prime \prime}\left(s_{0}(t)\right)\right] .
\end{aligned}
$$

It is convenient to recast Eqn (52) in the $y$ domain by defining the function $t_{0}(y)$ such that $t=t_{0}(y)$ is the solution of the equation $y=s_{0}(t)$, i.e. $t=t_{0}\left(s_{0}(t)\right)$ or $y=s_{0}\left(t_{0}(y)\right)$. In effect, $t_{0}(y)$ is the time at which the mean melt front reaches the location $y$.

Using this notation, Eqn (52) takes the form

$$
\begin{gathered}
\frac{1}{2 m^{2}}\left(m y w^{2}-w-m y\right) f^{\prime \prime}(y)+\frac{1}{m} f^{\prime}(y)-\frac{1}{2}\left(m y w^{2}+w-m y\right) f(y) \\
=-\frac{A}{m^{3}} \dot{s}_{1}\left(t_{0}(y)\right)\left[1-\frac{1}{\cosh (m y)}\right]-\frac{A}{m^{2}} \dot{s}_{0}\left(t_{0}(y)\right) s_{1}\left(t_{0}(y)\right) w
\end{gathered}
$$

where $w=\tanh \left(m s_{0}\left(t_{0}(y)\right)\right)=\tanh (m y)$, and $\dot{s}_{0}\left(t_{0}(y)\right), \dot{s}_{1}\left(t_{0}(y)\right), s_{1}\left(t_{0}(y)\right)$ are the values of the respective quantities at the instant when the mean melt front reaches the location $y$.

Finally, we determine $P_{1}(t) \cos (m x)$, the perturbation in contact pressure about its mean value at the casting/mold interface, from Eqns (25), (41), (48) with the result

$$
P_{1}(t)=-A\left[\dot{s}_{0}(t) s_{1}(t)+\dot{s}_{1}(t) s_{0}(t)\right]+m^{2} a_{1}(t)
$$

where $A, a_{1}(t)$ can be found from (33), (53) and we have imposed the condition $f(0)=0$. This can be done without loss of generality since the solution of Eqn (55) includes constants which govern the apportionment of a time-independent biharmonic term between $\Phi_{1}, \Phi_{2}$ (see Section 2) and which can be assigned arbitrarily.

\subsection{Richmond's problem}

The problem considered by Richmond et al. [12] is a special case of the above analysis in which the prescribed heat flux is constant, i.e.

$$
Q(x, t)=Q_{0}+Q_{1} \cos (m x)
$$

where $Q_{0}$ and $Q_{1}$ are time-independent constants. From Eqns (15), we then have

$$
s_{0}(t)=\frac{Q_{0} t}{L \rho} ; \quad s_{1}(t)=\frac{Q_{1} t}{L \rho}
$$


where we have used the initial condition $s(x, 0)=0$ to determine the constants of integration.

These results can be used to simplify the right-hand side of Eqn (55), but the resulting equation cannot be solved in closed form because of the hyperbolic functions in the coefficients. However, an approximate solution is possible for the early stages of solidification for which $m s_{0}(t) \ll 1$. It then follows that $m y \ll 1$ and we can approximate $w[=\tanh (m y)]$ and $\cosh (m y)$ as power series truncated to terms in $(m y)^{3}$ and below.

With this simplification, Eqn (55) reduces to

$$
y f^{\prime \prime}(y)-f^{\prime}(y)=\frac{3}{2} \frac{A Q_{1}}{L \rho} y^{2}
$$

which has the solution

$$
f(y)=\frac{1}{2} \frac{A Q_{1}}{L \rho} y^{3} .
$$

Using the same approximation in Eqn (53), we obtain

$$
a_{1}(t)=\frac{A Q_{1} Q_{0} t}{(m L \rho)^{2}}\left[2+\frac{1}{6}\left(\frac{m Q_{0} t}{L \rho}\right)^{2}\right]
$$

and hence, from (33), (56), (61),

$$
P_{1}(t)=\frac{m^{2} E \alpha Q_{1}}{6(1-v) K}\left(\frac{Q_{0} t}{\rho L}\right)^{3} .
$$

This result is identical with that obtained by Richmond et al. [12] using a simple beam theory to describe the stresses in the solidified shell. In effect, truncation of the power series for the stress function $\Phi$ to four terms is equivalent to a derivation based on equilibrium alone (since no compatibility relations are then involved) and hence to a beam theory. Equation (62) indicates that the contact pressure increases from the onset of solidification in regions where the heat flux at the casting/mold interface is greatest, i.e. near $x=2 n \pi$. In the presence of a contact resistance which falls with pressure, this constitutes a system with positive feedback, which therefore has the potential for being unstable.

To examine the range of validity of Eqn (62) and hence of Richmond's beam solution, we have solved Eqn (55) numerically using the Runge-Kutta fourth-order method, without truncation of the hyperbolic series. The results are presented in Fig. 2 and show that the approximate result (62) is acceptable in the range $0<m s_{0}(t)<0.5$.

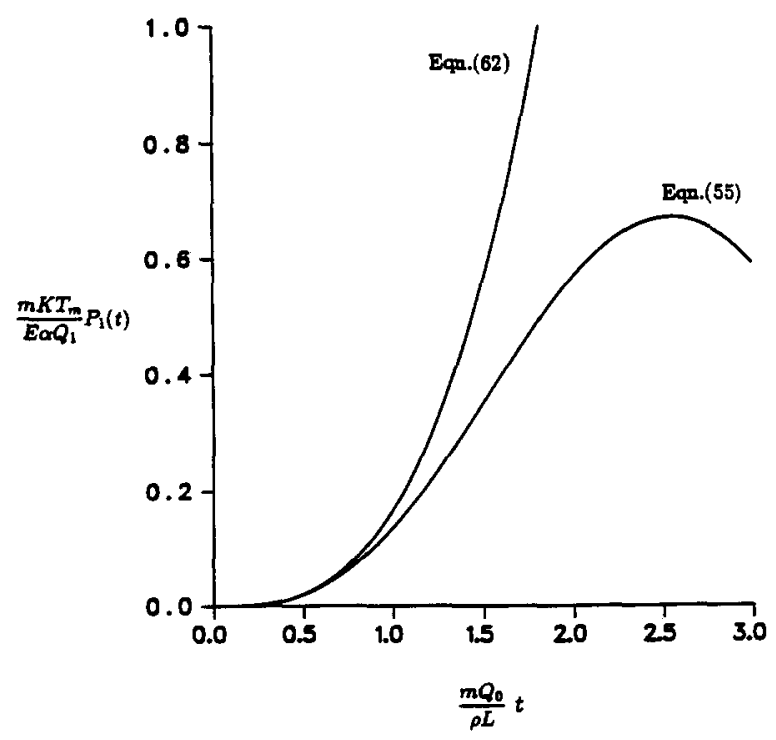

Fig. 2. Perturbation in contact pressure with time for constant heat flux at the mold. 


\subsection{Heat transfer across a thermal contact resistance}

We now consider the case in which the heat flux at the casting/mold interface is determined by conduction across a thermal contact resistance, $R(x, t)$, at the interface, which is assumed to be a continuous and differentiable function of the contact pressure, $P(x, t)$. We also assume that the temperature of the rigid plane mold at this interface is a constant which can be taken as zero without loss of generality.

From the definition of thermal resistance, the heat flux $Q(x, t)$ at the interface can therefore be written

$$
Q(x, t)=\frac{T(x, 0, t)}{R(P(x, t))}
$$

where $T(x, 0, t)$ is the temperature of the solidified layer adjacent to the mold.

3.4.1. The zeroth-order solution. The unperturbed or zeroth-order form of this relation is

$$
Q_{0}(t)=\frac{T_{0}(0, t)}{R_{0}}
$$

where the unperturbed contact resistance $R_{0} \equiv R\left(P_{0}\right)$ is independent of time, since the unperturbed contact pressure is always equal to the constant hydrostatic pressure $p$, as shown in Section 3.2.1 above.

Substituting for $Q_{0}(t), T_{0}(0, t)$ from Eqns (15), (18), respectively, and rearranging the terms, we obtain the equation

$$
L \rho \dot{s}_{0}(t)=\frac{T_{m}}{\left(R_{0}+s_{0}(t) / K\right)}
$$

from which

$$
s_{0}(t)=-K R_{0}+\left(K^{2} R_{0}^{2}+2 \frac{K T_{m}}{L \rho} t\right)^{1 / 2}
$$

and hence

$$
Q_{0}(t)=\frac{K T_{m}}{s_{0}(t)+K R_{0}} ; \quad T_{0}(y, t)=T_{m}\left[\frac{y+K R_{0}}{s_{0}(t)+K R_{0}}\right]
$$

from Eqns (15), (18).

This completes the solution of the unperturbed problem, for which the stress components can be recovered using (30), (31).

3.4.2. Perturbation of the resistance equation. We now use the technique developed in $[16,17]$ to obtain a relationship between the small perturbations $\Delta Q=Q_{1}(t) \cos (m x)$, $\Delta T=T_{1}(0, t) \cos (m x), \Delta P=P_{1}(t) \cos (m x)$ from the zeroth-order values $Q_{0}, T_{0}, R_{0}$. Differentiating Eqn (63), we find

$$
\frac{\Delta Q}{Q_{0}}=\frac{\Delta T}{T_{0}}-\frac{R^{\prime}\left(P_{0}\right) \Delta P}{R_{0}}
$$

where $R^{\prime}=\mathrm{d} R / \mathrm{d} P$.

Each of the perturbed quantities varies with $x$ only through the multiplier $\cos (m x)$, and hence Eqn (68) reduces to

$$
\frac{Q_{1}(t)}{Q_{0}}=\frac{T_{1}(0, t)}{T_{0}(0, t)}-\frac{R^{\prime}\left(P_{0}\right) P_{1}(t)}{R_{0}} .
$$

Substituting for $Q_{0}(t), T_{0}(0, t), Q_{1}(t), T_{1}(0, t)$ from (15), (19), (67) and rearranging the terms, we obtain

$$
\frac{L \rho}{K T_{m}}\left[s_{0}(t)+K R_{0}\right]^{2} \dot{s}_{1}(t)+s_{1}(t)+K R^{\prime}\left(P_{0}\right) P_{1}(t)=0
$$

where we have used Eqn (65) for $\dot{s}_{0}(t)$. 
Finally, substituting for $P_{1}(t)$ from Eqn (56), and using the notation $y=s_{0}\left(t_{0}(y)\right)$ introduced in Section 3.2.2, we have

$$
\begin{aligned}
K R^{\prime}\left(P_{0}\right) & \frac{m y}{2} \frac{\tanh (m y)}{\cosh (m y)} f^{\prime \prime}(y)-\frac{K R^{\prime}\left(P_{0}\right)}{\cosh (m y)}\left[1+\frac{m y}{2} \tanh (m y)\right] f(y) \\
= & \left\{K R^{\prime}\left(P_{0}\right) A\left[y-\frac{\tanh (m y)}{m}\right]-\left(y+K R_{0}\right)^{2} \frac{L \rho}{K T_{m}}\right\} \dot{s}_{1}\left(t_{0}(y)\right) \\
& +\left\{K R^{\prime}\left(P_{0}\right) A \frac{K T_{m}}{L \rho\left(y+K R_{0}\right)}\left[1-\frac{1}{\cosh (m y)}\right]-1\right\} s_{1}\left(t_{0}(y)\right) .
\end{aligned}
$$

Equations (55), (71) then constitute a pair of coupled ordinary differential equations to determine the unknown quantities $f(y), s_{1}(t)$.

\section{SOLUTION AND RESULTS}

Before proceeding to the solution for $f(y), s_{1}(t)$, it is convenient to introduce the dimensionless variables

$$
\begin{aligned}
\tau & =m^{2} \frac{K T_{m}}{L \rho} t \\
\eta & =m y=m s_{0}\left(t_{0}(y)\right) \\
\lambda(\tau) & =m s_{1}(t) \\
F(\eta) & =\frac{m^{2}(1-v)}{E \alpha T_{m}} f(y) \\
\bar{P} & =\frac{(1-v)}{E \alpha T_{m}} P \\
\bar{R}_{0} & =m K R\left(P_{0}\right)=m K R_{0} .
\end{aligned}
$$

Following the notation $t_{0}(y)$ introduced in Section 3.2.2, we define $\tau_{0}(\eta)$ as the dimensionless time at which the mean melt front reaches the location $\eta$. We also define $\bar{R}^{\prime}=\bar{R}^{\prime}\left(\bar{P}_{0}\right)$ where $\bar{P}_{0}$ is the dimensionless unperturbed contact pressure.

Equations (55), (71) then become

$$
\begin{aligned}
& \frac{1}{2}\left[\eta \tanh ^{2}(\eta)-\tanh (\eta)-\eta\right] F^{\prime \prime}(\eta)+F^{\prime}(\eta) \\
& -\frac{1}{2}\left[\eta \tanh ^{2}(\eta)+\tanh (\eta)-\eta\right] F(\eta) \\
& =-\left[1-\frac{1}{\cosh (\eta)}\right] \lambda^{\prime}\left(\tau_{0}(\eta)\right)-\frac{\tanh (\eta)}{\left(\eta+\bar{R}_{0}\right)} \lambda\left(\tau_{0}(\eta)\right)
\end{aligned}
$$

and

$$
\begin{aligned}
\bar{R}^{\prime} \frac{\eta}{2} \frac{\tanh (\eta)}{\cosh (\eta)} & F^{\prime \prime}(\eta)-\frac{\bar{R}^{\prime}}{\cosh (\eta)}\left[1+\frac{\eta}{2} \tanh (\eta)\right] F(\eta) \\
= & \left\{\bar{R}^{\prime}[\eta-\tanh (\eta)]-\left(\eta+\bar{R}_{0}\right)^{2}\right\} \lambda^{\prime}\left(\tau_{0}(\eta)\right) \\
& +\left\{\frac{\bar{R}}{\eta+\bar{R}_{0}}\left[1-\frac{1}{\cosh (\eta)}\right]-1\right\} \lambda\left(\tau_{0}(\eta)\right) .
\end{aligned}
$$

These two coupled equations serve to determine the functions $F(\eta), \lambda(\tau)$, given suitable initial conditions at $\eta=0$. They are both second-order ordinary differential equations with variable coefficients, but they can be reduced to a single differential equation as shown in the next section. 


\subsection{Reduction to a single equation}

We first consider the simpler case of a pair of first order equations with variable coefficients in the form

$$
\begin{aligned}
& b_{0}(y) G^{0}(y)+b_{1}(y) G^{1}(y)=c_{0}(y) H^{0}(y)+c_{1}(y) H^{1}(y)+h_{1}(y) \\
& d_{0}(y) G^{0}(y)+d_{1}(y) G^{1}(y)=e_{0}(y) H^{0}(y)+e_{1}(y) H^{1}(y)+h_{2}(y)
\end{aligned}
$$

where $G^{i}(y) \equiv \mathrm{d}^{i} G / \mathrm{d} y^{i}, H^{i}(y) \equiv \mathrm{d}^{i} H / \mathrm{d} y^{i}, G, H$ are two unknown functions of $y$ and the lower-case symbols denote known functions of $y$.

Treating $y$ as a parameter and $G^{0}, G^{1}$ as independent functions, we can solve (80), (81) as a pair of simultaneous equations whose solution gives $G^{0}, G^{1}$ as linear functions of $H^{0}, H^{1}$ with $y$-dependent coefficients. Substituting this solution into the so far unused relation $G^{1}=\mathrm{d} G^{0} / \mathrm{d} y$ then yields a single second-order ordinary differential equation for $H$ with variable coefficients.

The same technique can be adapted to the more general equations

$$
\begin{aligned}
& \sum_{i=0}^{n} b_{i}(y) G^{i}(y)=\sum_{i=0}^{m} c_{i}(y) H^{i}(y)+h_{1}(y) \\
& \sum_{i=0}^{n} d_{i}(y) G^{i}(y)=\sum_{i=0}^{m} e_{i}(y) H^{i}(y)+h_{2}(y) .
\end{aligned}
$$

For example, if we carry all terms $i \leqslant 2$ to the right-hand side of the equations, we can solve for $G^{n}, G^{n-1}$ and obtain an equation of order $n-1$ in $G$ by substituting into the relation $G^{n}=\mathrm{d} G^{n-1} / \mathrm{d} y$. The resulting equation and the solution for $G^{n-1}$ now constitute a new pair of equations of degree $(n-1)$ in $G$ and $(m+1)$ in $H$. The procedure can therefore be used recursively until a single equation of degree $(m+n)$ in $H$ only is obtained.

This procedure was used to eliminate the residual stress function ${ }^{\dagger} F(\eta)$ from Eqns (78), (79). In principle this gives a third-order equation for $\lambda$, but in the event the coefficient of $\lambda^{\prime \prime \prime}$ proved to be identically zero leaving a second-order ordinary differential equation which was solved by the Runge-Kutta fourth-order method.

The initial value $\lambda(0)$ must be taken as zero to satisfy $(12)$, since $s_{0}(0)=0$. An initial perturbation of the system can then be introduced by giving a small initial value to $\lambda^{\prime}(0)$. Once $\lambda$ has been determined, the residual stress function $F(\eta)$ can be recovered by numerical solution of Eqn (78) and the perturbation in contact pressure $P_{1}(t)$ is given by (56).

\subsection{Numerical results}

The governing second-order differential equation is linear and homogeneous and has only one non-zero initial condition - the value of $\lambda^{\prime}(0)$. It follows that for given values of the dimensionless parameters $\bar{R}_{0}, \bar{R}^{\prime}$, the solution for $\lambda$ is linearly proportional to $\lambda^{\prime}(0)$. In all the following results, the initial value, $\lambda^{\prime}(0)$, was taken as 0.1 .

Figure 3 shows the development of the perturbation in the solidification front with time for $\bar{R}_{0}=0.3$ and various values of $\bar{R}^{\prime}$. Notice that we anticipate that the resistance will fall with increasing contact pressure and hence that realistic values of $\bar{R}^{\prime}$ are negative. As we would expect, the perturbation levels off to a constant value when $\bar{R}^{\prime}$ is small-i.e. when the resistance is relatively insensitive to pressure. However, for large values of $\bar{R}^{\prime}$, indicating a significant pressure dependence, the perturbation grows in a quasi-exponential fashion.

To examine the effect of the mean value of contact resistance $\bar{R}_{0}$, and hence of the mean contact pressure, $p$, we plot the development of $\lambda(\tau)$ for $\bar{R}^{\prime}=-300$ and various $\bar{R}_{0}$ in Fig. 4. Generally, low values of $\bar{R}_{0}$ conduce to more rapid growth of the perturbation, though this behavior only becomes apparent after an initial period. We should note that a low value of $\bar{R}_{0}$ will also increase the velocity of the unperturbed unidirectional process as indicated by Eqn (65), so that a more meaningful measure of the severity of the instability is obtained by eliminating $\tau$ and plotting $\lambda=m s_{1}$ as a function of $m s_{0}$. This is shown in Fig. 5

\footnotetext{
${ }^{\dagger}$ It might seem more efficient to eliminate $\lambda$, since Eqns (78), (79) are only of first order in this function. However, it is then difficult to decide on appropriate initial conditions for $F(\eta)$, whereas the initial conditions for the perturbation in the solidification front, $\lambda$, can be determined from physical considerations.
} 


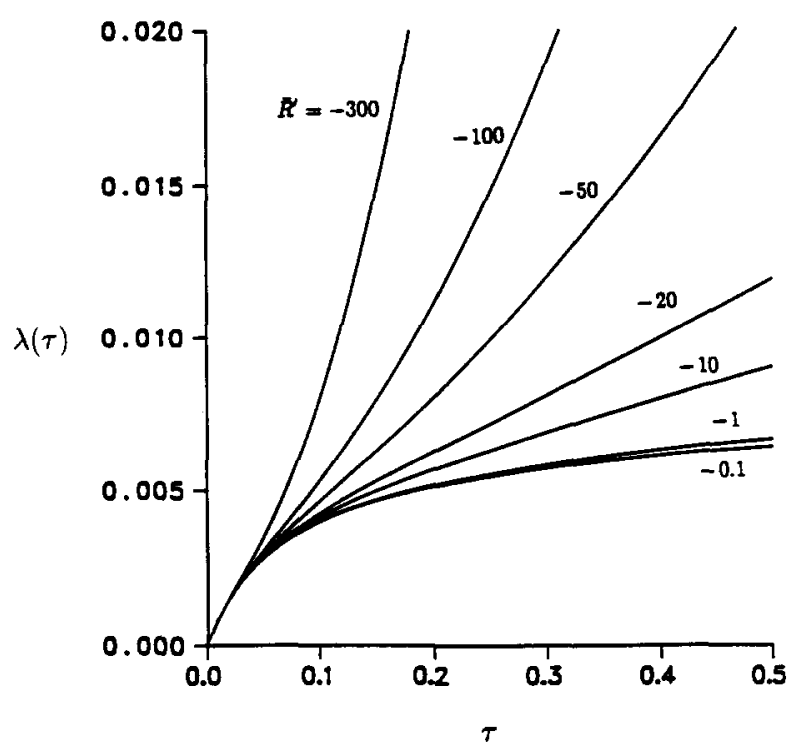

Fig. 3. Perturbation in solidification front with time for $\bar{R}_{0}=0.3$ and various values of $\bar{R}^{\prime}$.

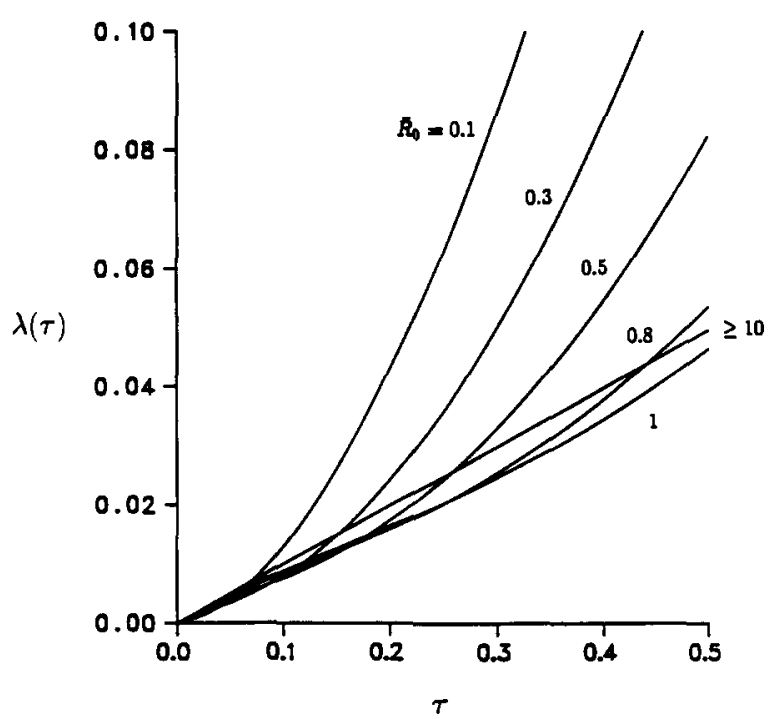

Fig. 4. Perturbation in solidification front with time for $\bar{R}^{\prime}=-300$ and various values of $\bar{R}_{0}$.

for $\bar{R}^{\prime}=-300$ and various values of $\bar{R}_{0}$. The various curves in this figure are plotted for a constant initial value of $\dot{s}_{1} / \dot{s}_{0}(=0.01)$. This would be the case if, for example, the mold temperature had a small spatially-sinusoidal perturbation of given amplitude. The results confirm that low values of $\bar{R}_{0}$ favor the growth of the perturbation.

Finally, we investigate the effect of wavelength on the growth rate of the perturbation for fixed values of $\bar{R}_{0}, \bar{R}^{\prime}$. For this purpose it is convenient to define the new dimensionless parameters

$$
\begin{aligned}
l & =\frac{\lambda(\tau)}{\bar{R}_{0}}=\frac{s_{1}(t)}{K R_{0}} \\
\theta & =\frac{\lambda(\tau)}{\bar{R}_{0}^{2}}=\frac{T_{m}}{L \rho K R_{0}^{2}} t \\
\bar{R}_{m}^{\prime} & =\frac{\bar{R}^{\prime}}{\bar{R}_{0}}=\frac{E \alpha T_{m}}{(1-v) R_{0}} R^{\prime}\left(P_{0}\right)
\end{aligned}
$$




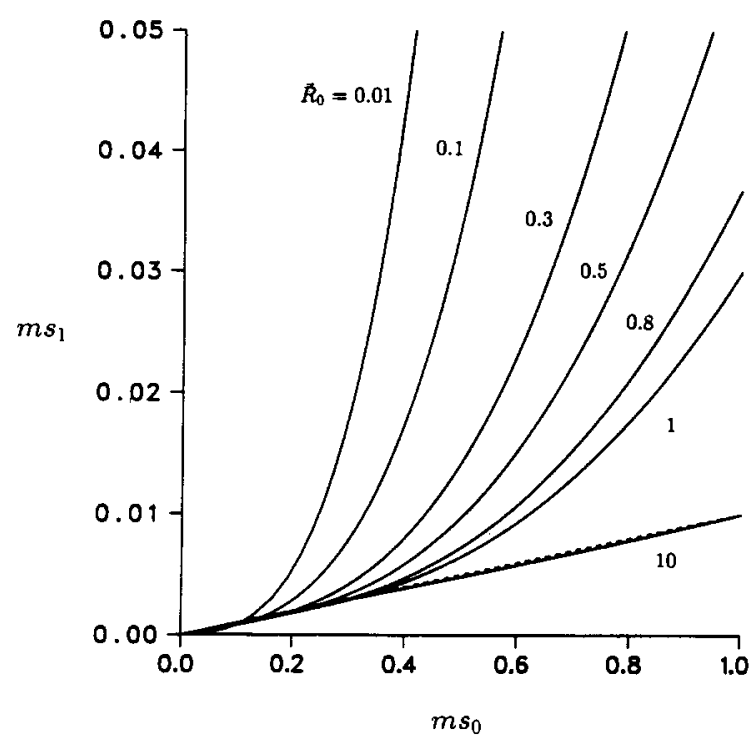

FIG. 5. Perturbation in solidification front as a function of mean front position for $\bar{R}^{\prime}=-300$ and various values of $\bar{R}_{0}$.

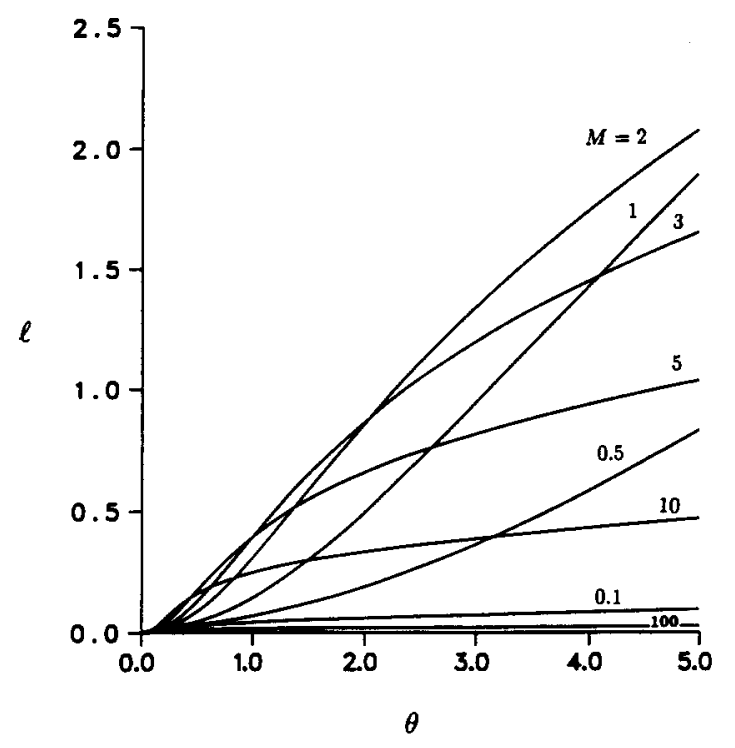

FIG. 6. Effect of wavelength on the growth of the perturbation for $\bar{R}_{m}^{\prime}=-300$.

which are chosen to be independent of $m$ when the other physical parameters are held constant. The effect of varying $m$ will appear through the variation in the parameter $\bar{R}_{0}=m K R_{0}$, which we here rename $M$ to highlight its present function of characterizing the wavenumber of the perturbation.

Figure 6 shows the variation of the perturbation amplitude $l(\theta)$ as a function of time, $\theta$, for $\bar{R}_{m}^{\prime}=-300, l^{\prime}(0)=0.1$, and various wavenumbers $M$. In general, large values of $M$, corresponding to short wavelengths, peak early and then level out, whereas the longer wavelengths (small $M$ ) peak later and reach larger amplitudes. Assuming a random distribution of wavelengths in the initial perturbation, we should therefore anticipate larger wavelengths coming to dominate the instability as solidification proceeds.

\section{CONCLUSIONS}

The above results demonstrate that a small spatially-sinusoidal perturbation in nominally unidirectional solidification can grow rapidly as a result of interaction between thermo- 
elastically-induced variations in contact pressure and the thermal contact resistance at the mold/casting interface if this resistance is sufficiently pressure-sensitive. This mechanism therefore provides a plausible explanation of non-uniformities observed in some interrupted solidification experiments.

The assumptions made in the analysis are clearly very restrictive-notably the small Stefan number approximation and the assumptions that the mold is rigid and that the constitutive behavior of the solidified material is elastic. Previous analyses of the stability of thermoelastic contact between two solids [17] suggest that the thermoelastic distortion of the mold would be conducive to greater instability of the process. On the other hand, plastic deformation of the solidified shell will tend to relax the thermoelastic stresses and may have a stabilizing effect. These topics require a more sophisticated model of the process and will be addressed in future investigations.

Acknowledgements-The authors gratefully acknowledge the support of the National Science Foundation under Contract No. MSM-8820623.

\section{REFERENCES}

1. A. M. Clausing and B. T. ChaO, Thermal contact resistance in a vacuum environment. ASME J. Heat Transfer 87, 243 (1965).

2. T. R. Thomas and S. D. Probert, Thermal contact resistance: The directional effect and other problems. Int. J. Heat Mass Transfer 13, 789 (1970).

3. Y. P. ShlYKov and Y. A. GaNin, Thermal resistance of metallic contacts. Int. J. Heat Mass Transfer 7, 921 (1964).

4. M. G. Cooper, B. B. Mikic and M. M. Yovanovich, Thermal contact conductance. Int. J. Heat Mass Transfer 12, 279 (1969).

5. J. A. Greenwood and J. B. P. Williamson, The contact of nominally flat surfaces. Proc. R. Soc. (London) A295, 300 (1966).

6. K. Ho and R. D. PEHLKe, Metal-mold interfacial heat transfer. Metall. Trans. 16B, 585 (1985).

7. K. Ho and R. D. PeHLKE, Mechanisms of heat transfer at a metal-mold interface. AFS Trans. 92,587 (1984).

8. O. Richmond and N. C. HuANG, Interface stability during unidirectional solidification of a pure metal. Proc. 6th Canadian Congress of Applied Mechanics, Vancouver, pp. 453-454 (1977).

9. NAI-Yi Li and J. R. BARBER, Sinusoidal perturbation solutions for planar solidification. Int. J. Heat Mass Transfer 32, 935 (1989).

10. P. J. WraY, Non-uniform growth of a plate on a chilled surface. Presented at the Annual Meeting of A.I.M.E., Atlanta, Georgia. (6-10 March 1977).

11. L. G. HeCtor, Private communication.

12. O. Richmond, L. G. HeCTOR and J. M. FrIDY, Growth instability during nonuniform directional solidification of pure metals. J. Appl. Mech. 57, 529 (1990).

13. H. M. WestergaARD, Theory of Elasticity and Plasticity, Art. 64. Dover, New York (1964).

14. O. RICHMOND and R. H. TIEN, Theory of thermal stresses and air-gap formation during the early stages of solidification in a rectangular mold. J. Mech. Phys. Solids 19, 273 (1971).

15. NAI-YI Li and J. R. Barber, Residual stresses in castings with axisymmetric solidification. Transport Phenomena in Manufacturing, pp. 27-34. ASME, New York (1989).

16. J. R. BARBER, J. DUNDURS and M. ComNINOU, Stability considerations in thermoelastic contact. J. Appl. Mech. 47, 871 (1980).

17. R. ZHANG and J. R. BARBER, Effect of material properties on the stability of static thermoelastic contact. J. Appl. Mech. 57, 365 (1990). 